\title{
Perfusion-weighted magnetic resonance imaging in the assessment of haemodynamics following stent angioplasty in patients with symptomatic middle cerebral artery plaque stenosis at the M1 segment
}

\author{
YUN-CAI RAN ${ }^{1}$, MING ZHU $^{2}$, YONG ZHANG ${ }^{1}$, TENG-FEI LI ${ }^{2}$ and JING-LIANG CHENG ${ }^{1}$ \\ Departments of ${ }^{1}$ Magnetic Resonance and ${ }^{2}$ Interventional Therapy, \\ The First Affiliated Hospital of Zhengzhou University, Zhengzhou, Henan 450052, P.R. China
}

Received December 29, 2015; Accepted February 6, 2017

DOI: $10.3892 / \mathrm{etm} .2017 .4747$

\begin{abstract}
The most effective strategy to assess changes in the brain haemodynamics of stent angioplasty in patients with symptomatic ischemia of the M1 segment of the middle cerebral artery (MCA) remains unknown. The purpose of the present study was to use perfusion-weighted magnetic resonance imaging (PWI) to evaluate the effect of stent angioplasty in treating patients with symptomatic MCA plaque stenosis. Stent angioplasty was performed on 23 patients with reduplicative transient ischaemic attack who were refractory to medical therapy. All patients had MCA plaque stenosis at the M1 segment. Brain PWI was obtained from four major regions of interest (ROIs) at the frontal parietal, temporal, lateral ventricle and basal ganglia lobes prior to and following stent implantation. In addition, cerebral blood flow (CBF), cerebral blood volume, mean transit time (MTT) and time to peak (TTP) parameters derived from PWI were calculated. All patients underwent digital subtraction angiography following surgery to confirm the patency. Computed tomography angiography or PWI was performed 1 week and 3 months post-surgery. According to pre-operative PWI, there was significant hypoperfusion in the symptomatic frontal parietal, temporal, lateral ventricle and basal ganglia lobes. By contrast, the regional $\mathrm{CBF}$ and $\mathrm{CBF}$ increased in the ROIs of the affected cerebral hemisphere 3 months after stent implantation $(\mathrm{P}<0.05$ vs. pre-operative data). Additionally, post-operative MTT and TTP in the ROIs on the operative side were significantly shorter than pre-operative MTT and TTP $(\mathrm{P}<0.05)$. During the follow-up period, the frequency of transient ischaemic attack was reduced or disappeared in all patients during the
\end{abstract}

Correspondence to: Dr Jing-Liang Cheng, Department of Magnetic Resonance, The First Affiliated Hospital of Zhengzhou University, 1 East Road, Zhengzhou, Henan 450052, P.R. China E-mail: jingliangchengdoc@163.com

Key words: stent angioplasty, plaque stenosis, cerebral artery, ischaemic strokes follow-up. In conclusion, PWI enables an effective and objective assessment of haemodynamics prior to and following stent angioplasty in patients with plaque stenosis of MCA at the M1 segment.

\section{Introduction}

The most effective strategy to assess changes in brain haemodynamics of stent angioplasty in patients with symptomatic ischemia of the M1 segment of the middle cerebral artery (MCA) remains unknown. The high morbidity rates $(63 \%$ in low-income and middle-income countries) and mortality rates (57\% in low-income and middle-income countries) (1) of ischemic strokes are a global health concern. Intracranial arterial stenosis is an important cause of ischaemic stroke and the incidence of MCA stenosis in patients with stroke is $7.0-17.7 \%$ (2). Reportedly, the recurrence rate of symptomatic cerebrovascular stenosis (represented as $>70 \%$ stenosis) is $23 \%$ following treatment (3). However, there is controversy over what the most effective treatment for MCA stenosis is. The outcomes of medical treatment for patients with MCA stenosis are unfavorable (4). It has been demonstrated that medical treatment with warfarin or aspirin fails to prevent further ischaemic events (5). In recent years, the efficacy of intravascular stent angioplasty to treat carotid stenosis has improved and novel techniques have emerged that permit intracranial vascular stenting $(6,7)$. Despite its post-operative complication rate, intravascular stent angioplasty remains the primary surgical treatment option for patients receiving ineffective drug therapy or those with recurrent symptomatic MCA stenoses. Furthermore, intravascular stenting is currently the preferred preventative treatment and can significantly improve levels of local perfusion due to MCA stenosis, thereby preventing ischaemic strokes and significantly reducing the incidence of stroke $(8,9)$.

The haemodynamics of MCA stenosis are extraordinarily complex. Currently, there is no standard method of assessing the changes in brain haemodynamics that occur following stent angioplasty in patients with MCA atherosclerotic stenosis at the M1 segment. Digital subtraction angiography (DSA) is the gold standard for diagnosing intracranial arterial 
stenosis; however, it is expensive, invasive and unable to detect haemodynamic changes. Therefore, DSA cannot be executed within a short time frame and cannot be used for follow-up testing following stent placement (10). Due to developments in medical imaging technology, various methods exist to study the haemodynamics of these patients, comprising computed tomographic perfusion (11), positron emission tomography (PET) and single photon emission computed tomography (SPECT) (12). Nevertheless, these methods may potentially cause radiation damage and other limitations such as long scanning duration and highly allergenic (due to the use of iodine as a contrast agent) $(11,12)$.

Perfusion-weighted magnetic resonance imaging (PWI) is a relatively novel imaging technique that can detect the most important parameters of cerebral dynamics, including cerebral blood flow (CBF), cerebral blood volume (CBV), mean transit time (MTT) and time to peak (TTP) in a non-invasive manner $(13,14)$. PWI has been used to evaluate the changes of haemodynamics in patients with plaque stenosis of the MCA at the M1 segment; however, only a small amount articles have reported using PWI for the contrast of preoperative and postoperative collateral circulation (15). In the present study, cerebral haemodynamics were examined in patients with MCA stenosis at the M1 segment prior to and following stent angioplasty in order to determine its effectiveness, and to examine the clinical utility of using PWI to evaluate the effectiveness of stent angioplasty.

\section{Materials and methods}

Patients and clinical processes. High-resolution patch analysis was performed to remove arterial stenosis that was not caused by atherosclerosis. The present study retrospectively assessed 23 patients with symptomatic MCA atherosclerotic stenosis at the M1 segment. All the patients had to be over 18 years old suffering symptomatic MCA stenosis at the M1 segment. All patients suffered recurrent transient ischaemic attack (TIA) with repeated TIA during stroke treatment. Patients underwent segmentation using enterprise stents, which had a good result (the recurrent TIA attack was reduced or disappeared, no high perfusion syndrome and complication following post-operation follow up at 3 months) at The First Affiliated Hospital of Zhengzhou University between January 2012 and March 2015. The patients comprised of 11 females and 12 males with a median age of 57.8 years old (between 38 and 64 years old). All patients suffered recurrent transient ischaemic attack (TIA) despite receiving warfarin (9 patients: 6 females and 3 males) or aspirin medication (14 patients: 5 females and 9 males), and there were 4 patients ( 3 males and 1 females) with repeated TIA during stroke treatment. Computed tomography angiography (CTA) or magnetic resonance angiography supported MCA stenosis at the M1 segment and high resolution magnetic resonance imaging (MRI) confirmed that M1 segment stenosis was caused by local artery plaque formation. At the same time, the course of cerebral infarction in patients lasts for $>14$ days and 2 patients suffered ischemic stroke (mean onset time was 19.5 days) 1 female at day 22 and 1 male at day 17. The onset time for recurrent TIA attack was between 14 days and 6 months (mean 47.8 days), and all the patients had the enterprise stent used to perform angioplasty.
The calibre stenosis rate was $\geq 75 \%$ (between 75 and $90 \%$ ) with a median calibre stenosis rate of $84.9 \%$. Stent angioplasties were performed with 12 on the left side and 11 on the right side and pre-operative PWI images were obtained for all patients less than 1 week prior to surgery.

All patients underwent DSA following surgery in order to confirm the stent patency. They were monitored with PWI within the first week, 8 patients ( 3 females $/ 5$ males, mean age 55.3 years old) underwent testing with PWI at 3 months post-operation and the other 15 patients were assessed using computed tomography angiography (CTA) depending on the physician treating them. Following surgery, the symptoms disappeared in patients with recurrent TIA and there was no recurrence in the 3-month follow up period. All patients provided written informed consent to be included in the experiment and the present study was conducted in accordance with the declaration of Helsinki. Approval for the present study was granted by the Ethics Committee of The First Affiliated Hospital of Zhengzhou University (Zhengzhou, China).

PWI scan parameter. MRI was performed with a clinical 3.0-Tesla whole-body MR system (Verio-Tim, Siemens AG, Munich, Germany). PWI imaging (repetition time 1,500 msec, echo time $30 \mathrm{msec}$, flip angle $=90^{\circ}$, field of view $=230 \times 230 \mathrm{~mm}$, number of excitations $=1.00$ ) was performed following an injection $(3 \mathrm{ml} / \mathrm{sec}$ ) of gadopentetic acid diethylenetriaminepentaacetic acid (Magnevist ${ }^{\circledR}$, Bayer Healthcare Pharmaceuticals, Berlin, Germany; $0.1 \mathrm{mmol} / \mathrm{kg}$ ) with a high-pressure syringe needle through the ulnar vein. Images were generated from a $128 \times 128$ raw matrix following data acquisition. A slice thickness of $4.0 \mathrm{~mm}$ was used to obtain a high spatial resolution. Scanning time was $98 \mathrm{sec}$ with no interscan delay. Following scanning, the images were transferred onto a workstation (Syngo; Siemens AG) for data processing, which involved assessing the parameters of CBV, CBF, MTT and TTP from the pseudo-coloured picture of each selected lever. Region of interests (ROI) $5 \mathrm{~mm}$ in diameter were selected in the frontal parietal, temporal and lateral ventricles, and the basal ganglia areas avoiding blood vessels, cerebrospinal fluid and infarction regions. Simultaneously, the same location for each measurement was selected. Finally, the lesion contralateral region was selected to generate reference points, and the relative parameters; regional CBV (rCBV), CBF (rCBF), MTT (rMTT) and TTP (rTTP) were obtained from the comparison.

Changes in frequency of TIA attack. Changes in the frequency of TIA attack were felt by the patients themselves, so they were asked to indicate the frequency on day 14 pre-operation and week 1 and 3 months post-operation, the results of which were recorded. The unit of measure was/per individual/per day (/p/d).

Statistical analysis. All values are presented as the mean \pm standard deviation. Statistical analyses were performed with the paired-samples t-test and one-way analysis of variance. $\mathrm{P}<0.05$ was used to indicate a statistically significant difference.

\section{Results}

Changes in PWI pre-and post-operation. In total, 23 patients underwent stent angioplasty and all of them underwent PWI 
Table I. PWI analysis parameter analysis pre- and post-operation for ROIs of the ipsilateral operation hemisphere.

Post-operative

\begin{tabular}{lcccc} 
Ischemic hemisphere & PWI parameters & Pre-operative $(\mathrm{n}=23)$ & Week 1 $(\mathrm{n}=23)$ & Month 3 $(\mathrm{n}=8)$ \\
\hline ROIs & rCBV & $219.7 \pm 121.1$ & $208.3 \pm 86.2$ & $283.5 \pm 84.2^{\mathrm{b}, \mathrm{c}}$ \\
& rCBF & $40.3 \pm 26.5$ & $38.7 \pm 20.4$ & $55.1 \pm 21.0^{\mathrm{b}, \mathrm{c}}$ \\
& rMTT & $1,110.2 \pm 204.1$ & $542.6 \pm 107.2^{\mathrm{a}}$ & $526.0 \pm 99.3^{\mathrm{b}}$ \\
& rTTP & $2,093.1 \pm 126.2$ & $9,98.9 \pm 156.7^{\mathrm{a}}$ & $1,063.5 \pm 184.2^{\mathrm{b}}$
\end{tabular}

The ROIs of ischemic hemisphere were obtained from frontal parietal lobe, femporal lobe, fateral ventricle lober and basal ganglia lobe. $\mathrm{rCBV}$ in $\mathrm{ml} / 100 \mathrm{~g}$ tissue; $\mathrm{rCBF}$ in $\mathrm{ml} / 100 \mathrm{~g} / \mathrm{min}, \mathrm{rMTT}$ and $\mathrm{rTTP}$ in sec. $\mathrm{P}<0.05 \mathrm{vs}$. pre-operative PWI data. ${ }^{\mathrm{a}} \mathrm{P}<0.05$ pre-operation vs. $1 \mathrm{week}$ post-operation $\left({ }^{\mathrm{a}} \mathrm{P}_{\mathrm{rCBV}}=0.61,{ }^{\mathrm{a}} \mathrm{P}_{\mathrm{rCBF}}=0.34,{ }^{\mathrm{a} P \mathrm{PTT}}=0.021,{ }^{a} \mathrm{P}_{\mathrm{rTTP}}=0.001\right),{ }^{\mathrm{b}} \mathrm{P}<0.05$ pre-operation vs. 3 months post-operation $\left({ }^{\mathrm{b}} \mathrm{P}_{\mathrm{rCBV}}=0.001\right.$, $\left.\left.{ }^{b} \mathrm{P}_{\mathrm{rCBF}}=0.002\right),{ }^{b} \mathrm{P}_{\mathrm{rMTT}}=0.031,{ }^{\mathrm{b}} \mathrm{P}_{\mathrm{rTTP}}=0.001\right),{ }^{\mathrm{c}} \mathrm{P}<0.051$ week post-operation vs. 3 months post-operation $\left({ }^{\mathrm{c}} \mathrm{P}_{\mathrm{rCBV}}=0.001,{ }^{\mathrm{c}} \mathrm{P}_{\mathrm{rCBF}}=0.003,{ }^{\mathrm{c}} \mathrm{P}_{\mathrm{rMTT}}=0.36\right.$, $\left.{ }^{c} \mathrm{P}_{\mathrm{rTTP}}=0.47\right)$. PWI, perfusion-weighted magnetic resonance imaging; ROI, regions of interest; $\mathrm{rCBV}$, regional cerebral blood volume; rCBF, regional cerebral blood flow; rMTT, regional mean transit time; rTTP, regional time to peak.

$<1$ week prior to and 1 week following stent angioplasty. Of these patients, 8 underwent PWI and DSA 3 months following stent angioplasty (Table I). A total of 14 patients were selected for CTA to observe whether the blood vessels were smooth and imaging examination revealed that the stents of all the patients were unobstructed 3 months following stent angioplasty. In total, all 23 patients experienced TIA symptoms that disappeared post-operatively.

Table I presents the PWI parameter (rCBV, rCBF, rMTT and rTTP) analysis for different ROIs of the ipsilateral operation hemisphere pre- and post-operatively at different times. The results indicate that 23 patients experienced marked hypoperfusion in the ROIs of the ipsilateral cerebral hemisphere pre-operatively. When the pre- and post-operative PWI data were compared, the $\mathrm{rCBV}$ and $\mathrm{rCBF}$ in the ROIs of the operative hemisphere did not exhibit significant changes $(\mathrm{P}>0.05$ vs. pre-operative PWI data) 1 week later. However, 3 months following stent angioplasty, the rCBV and $\mathrm{rCBF}$ exhibited significant changes $(\mathrm{P}<0.05$ vs. pre-operative $\mathrm{PWI}$ data) compared with pre-operation data and data collected 1 week following surgery. rMTT and rTTP in the ROIs in the ischemic hemisphere were significantly shortened post-operatively compared with the pre-operative PWI data $(\mathrm{P}<0.05)$, however there were no significant changes in these regions between 1 week and 3 months post-operation.

In Figs. 1 and 2, the DSA and PWI images of 1 patient pre- and post-operatively were shared. The results indicate that MCA had a smooth appearance and a widened luminal diameter post-operatively compared with the MCA prior to the operation (Fig. 1). Furthermore, an improvement in perfusion was observed compared with prior to the operation (Fig. 2). These observations indicate that marked improvements in cerebral perfusion in the surgical hemisphere can be expected following stent angioplasty 3 months post-operation.

Changes in frequency of TIA attack. The numbers of TIA attack during 14 days pre-operation was $2.91 \pm 1.22 \mathrm{p} / \mathrm{d}$, which demonstrated significantly reduced numbers of TIA attack during 7 days post-operation, $0.49 \pm 0.24 \mathrm{p} / \mathrm{d}\left(\mathrm{P}_{1}=0.001 ; \mathrm{P}<0.05\right)$ and the numbers of TIA attack during 3 months post-operation was $0.37 \pm 0.12 \mathrm{p} / \mathrm{d}\left(\mathrm{P}_{2}=0.001 ; \mathrm{P}<0.05\right)$. The numbers of TIA attack during 7 days post-operation demonstrated no difference with the numbers of TIA attack during 3 months $\left(\mathrm{P}_{3}=0.80\right.$; $\mathrm{P}<0.05)$.

\section{Discussion}

It has been demonstrated that stent angioplasty is an effective therapy at restoring intracranial blood supply (16). However, as with other endovascular procedures, an effective and convenient method of assessing treatment efficacy and follow-up has not yet been developed. Furthermore, there is insufficient research available to detect the haemodynamic changes that occur following stent placement (10).

Multiple imaging techniques, including PET, SPECT, CT perfusion and PWI, are used to assess cerebral perfusion prior to and following cerebral revascularisation surgery. Each of these techniques has advantages and disadvantages (17). PET and SPECT have been widely used to assess cerebral perfusion $(12,18)$. Furthermore, PET and SPECT scanners are more expensive, and their clinical application are limited as the quantity of PET and SPECT scanners is lower than the number of MRI and CT scanners in hospitals and to their poor spatial resolution (19). Perfusion CT scanning is a relatively novel technique that measures cerebral haemodynamics and is very fast and available worldwide. Abe et al (12) used perfusion CT to assess the effect of percutaneous transluminal angioplasty by treating symptomatic MCA stenosis and the $\mathrm{CBF}$ exhibited changes at an early stage (5-20 days). However, CT has some disadvantages, including delay and dispersion of the contrast bolus, delivery of ionising radiation and the unsuitability of iodinated contrast material for certain patients (18). PWI as a various parameter and multi-level continuous imaging technology can assess the haemodynamic state of the brain tissue with a spatial resolution higher than that of other techniques. Furthermore, PWI detects changes in the homogeneity of local magnetic fields and provides information regarding brain perfusion in the microvascular bed. rCBF, rCBV, rMTT and rTTP can be measured by PWI and calculated by numerical integration. 


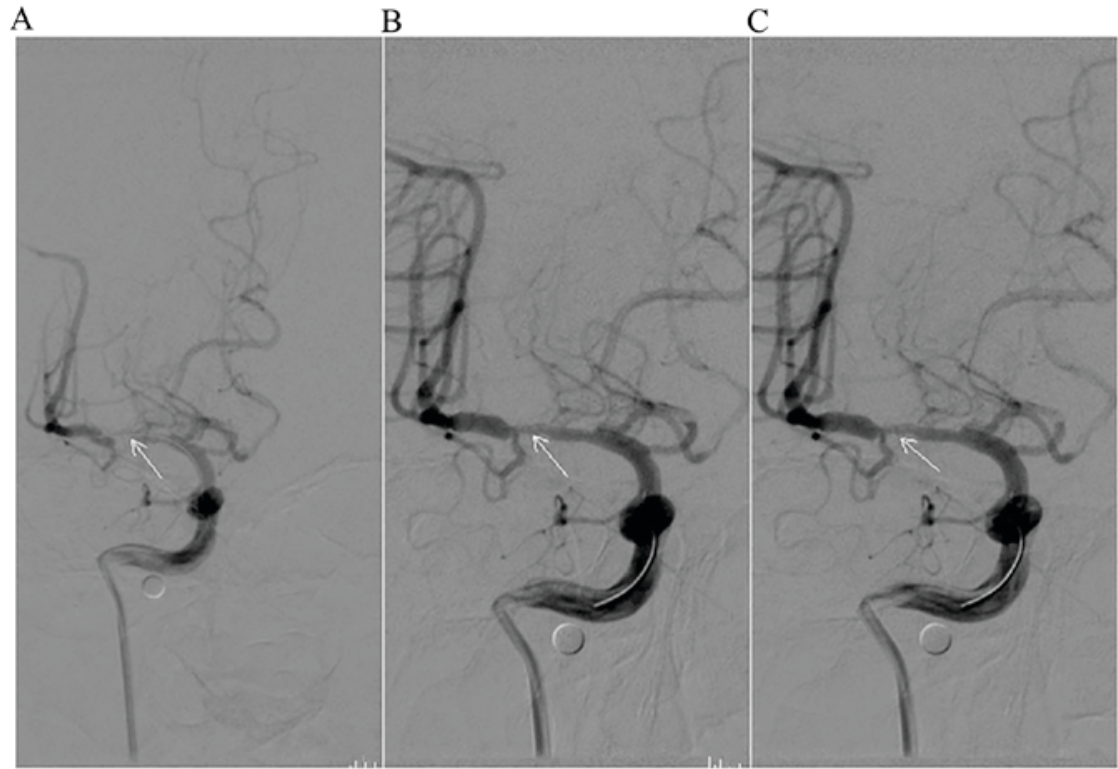

Figure 1. A representative case of a 41-year-old female patient who underwent right stent angioplasty due to a history of MCA atherosclerotic stenosis at the M1 segment. (A) Pre-operative right carotid digital subtraction angiography indicated that the M1 segment of the right MCA are occlusive performance (indicated by the arrow) and distal vessels were sparse. (B) Stent implantation and (C) 3 months following the operation, where stenosis was significantly improved at the M1 segment of MCA (indicated by the arrow) and the number of distal vessels were markedly increased compared with pre-operation. MCA, middle cerebral artery.
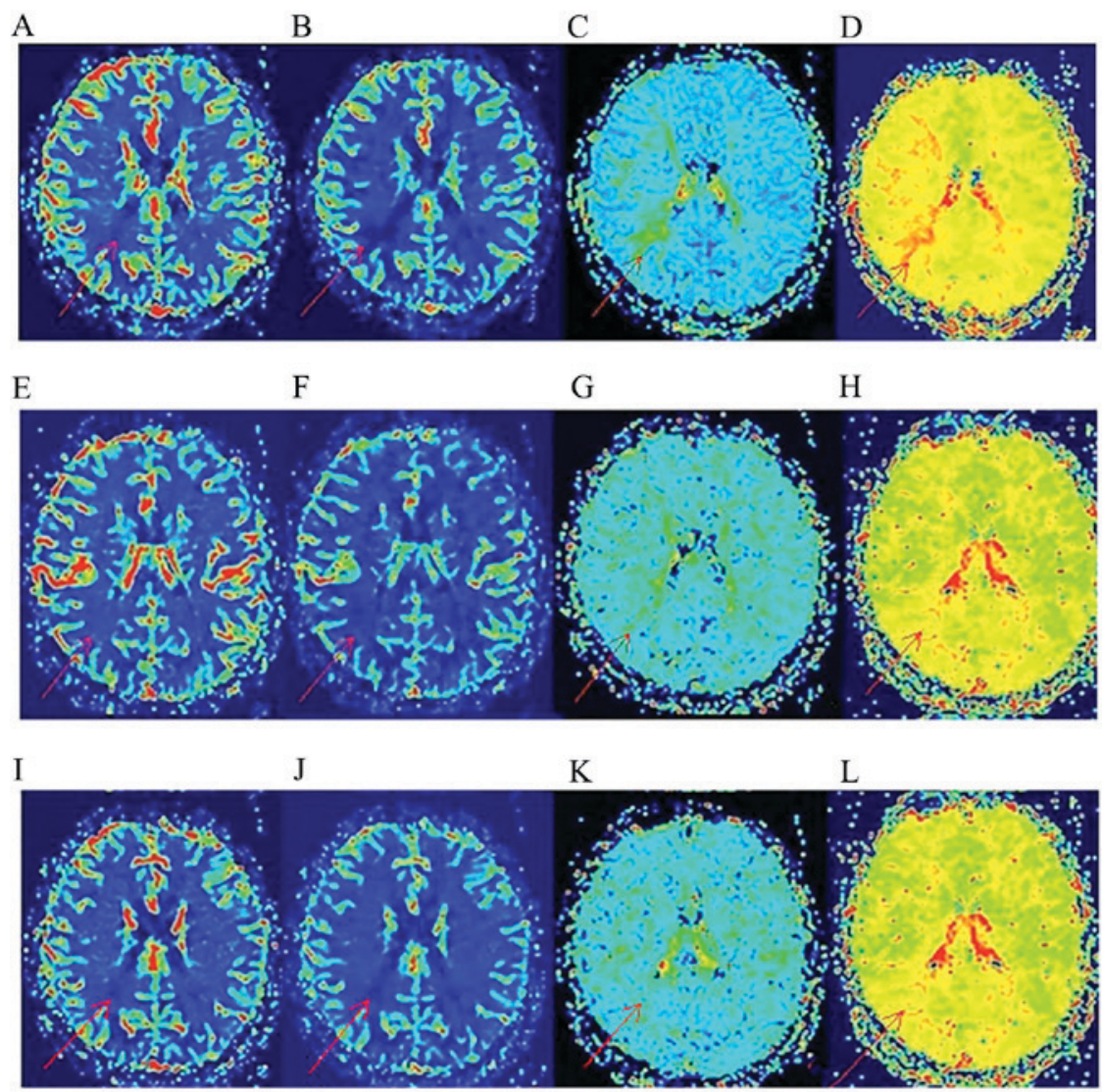

Figure 2. PWI images of a 41-year-old female patient who underwent right stent angioplasty due to a history of MCA atherosclerotic stenosis at the M1 segment. (A) CBV map pre-operation, (B) CBF map pre-operation, (C) MTT map pre-operation, (D) TTP map pre-operation, (E) CBV map 1 week post-operation, (F) CBF map 1 week post-operation, (G) MTT map 1 week post-operation, (H) TTP map 1 week post-operation, (I) CBV map 3 months post-operation, (J) CBF map 3 months post-operation, (K) MTT map 3 months post-operation, (L) TTP map 3 months post-operation. CBV and $\mathrm{CBF}$ maps exhibited little change (A and B) prior to and ( $\mathrm{E}$ and $\mathrm{F}$ ) following stent angioplasty; 3 months after post-operation, the ischemic range of the CBV map and CBF map changed between pre-operative (A and B) and 3 months later-post-operative (I and J). Ischemic range significantly reduced on the MTT and TTP maps prior to (C and D) and following ( $\mathrm{G}$ and $\mathrm{H})$ stent angioplasty and regions of ischemia did not exhibit any marked changes between a week post-operation $(\mathrm{G}$ and $\mathrm{H}$ ) and 3 months post-operation (K and L). PWI, perfusion-weighted magnetic resonance imaging; $\mathrm{CBV}$, cerebral blood volume; $\mathrm{CBF}$, cerebral blood flow; MTT, mean transit time; TTP, time to peak; MCA, middle cerebral artery. 
In addition, they can provide important information on the cerebral tissue contained in the ROIs (20).

The parameters of $\mathrm{rCBV}, \mathrm{rCBF}, \mathrm{rMTT}$ and $\mathrm{rTTP}$ exhibited different sensitivity and specificity to ischaemia, with rMTT being more sensitive to the scope of the ischaemic lesion compared with $\mathrm{rCBV}$ and $\mathrm{rCBF}$, which were impacted by collateral circulation (21). The peak time of the contrast agent injection was inversely proportional to the degree of stenosis and therefore, rTTP was also a sensible index for the low perfusion region (22). Therefore, the rMTT and rTTP parameters are able to identify the low perfusion region earlier than $\mathrm{rCBV}$ and $\mathrm{rCBF}$ which are more useful in determining the changes in cerebral haemodynamics. It has been determined that the extension degrees of rMTT and rTTP are positively correlated with the degree of arterial stricture $(23,24)$. Cerebrovascular stenosis led to the collateral circulation time of the blood flow through the narrow blood vessels and local being significantly longer than the time of the blood flow through the normal blood vessels and local collateral circulation prior to stent angioplasty. Furthermore, vascular stenosis was relieved following post-operative stent angioplasty. Initially, 1 week following operation, the rCBV and $\mathrm{rCBF}$ in the ROIs of the operative hemisphere did not differ significantly compared with the data collected prior to the operation. By contrast, rMTT and rTTP in the ROIs were significantly shortened 1 week following the operation compared with the pre-operative PWI data $(\mathrm{P}<0.05)$. This is similar to the results obtained by Gauvrit et al (25) and Wilkinson et al (26). Subsequently, the rCBV and rCBF of 3 months post-operation angioplasty significantly increased compared with the pre-operation $\left(\mathrm{P}_{\mathrm{rCBV}}=0.001, \mathrm{P}_{\mathrm{rCBF}}=0.002\right.$; $\mathrm{P}<0.05$; Table I) and 1 week post-operation $\left(\mathrm{P}_{\mathrm{rCBV}}=0.001\right.$, $\mathrm{P}_{\mathrm{rCBF}}=0.003$; $\mathrm{P}<0.05$; Table I). However, the $\mathrm{rCBV}$ and $\mathrm{rCBF}$ of pre-operation and 1 week post-operation demonstrated little difference $\left(\mathrm{P}_{\mathrm{rCBV}}=0.61, \mathrm{P}_{\mathrm{rCBF}}=0.34 ; \mathrm{P}<0.05\right.$; Table I $)$, which was consistent with the results of a study by Bendok et al (27). This result may be explained by the fact that the recoveries of the vascular bed in the blood supply area of the narrow artery is a lengthy process and no changes are evident in the short-term. rMTT and rTTP did not differ significantly between 1 week and 3 months post-operation. The result about changes in frequency of TIA attack showed difference with the changes of rCBV and rCBF; but it was consistent with rMTT and rTTP. That might be the vascular stenosis had been improved post-operation and the blood supply of the brain has basically met their own needs and showed little difference at 1 week and 3 months post-operation.

In conclusion, the present study used PWI to demonstrate the favorable outcome of performing a successful stent angioplasty by evaluating haemodynamics pre- and post-operatively in patients with MCA atherosclerotic stenosis at the M1 segment. Haemodynamic studies obtained from PWI revealed a significant improvement in cerebral perfusion following stent angioplasty. The present study demonstrates that PWI is a non-invasive, more convenient and less expensive technique compared with other imaging techniques and can provide quantitative evaluations of cerebral perfusion prior to and following stent angioplasty. The current study had some limitations, the primary limitation being that the number of cases included in the present study was relatively inadequate, some patients selected CTA at 3 months post-operation and there were differences in medical treatment. At the same time, patients with high perfusion post-operation were not included in the study, so there may be a deviation of the results' evaluation. Future studies should continue to collect the relevant cases including patients that suffer high perfusion post-operation to improve this study.

\section{References}

1. Krishnamurthi RV, Feigin VL, Forouzanfar MH, Mensah GA, Connor M, Bennett DA, Moran AE, Sacco RL, Anderson LM, Truelsen T, et al: Global and regional burden of first-ever ischaemic and haemorrhagic stroke during 1990-2010: Findings from the Global Burden of Disease Study 2010. Lancet Glob Health 1: e259-e281, 2013.

2. Wong KS, Li H, Lam WW, Chan YL and Kay R: Progression of middle cerebral artery occlusive disease and its relationship with further vascular events after stroke. Stroke 33: 532-536, 2002.

3. Zaidat OO, Klucznik R, Alexander MJ, Chaloupka J, Lutsep H, Barnwell S, Mawad M, Lane B, Lynn MJ and Chimowitz M; NIH Multi-center Wingspan Intracranial Stent Registry Study Group: The NIH registry on use of the wingspan stent for symptomatic 70-99\% intracranial arterial stenosis. Neurology 70: 1518-1524, 2008.

4. Chang YH, Hwang SK and Kwon OK: Primary angioplasty for symptomatic atherosclerotic middle cerebral artery stenosis. J Cerebrovasc Endovasc Neurosurg 16: 166-174, 2014.

5. Chimowitz MI, Lynn MJ, Howlett-Smith H, Stern BJ, Hertzberg VS, Frankel MR, Levine SR, Chaturvedi S, Kasner SE, Benesch CG, et al: Comparison of warfarin and aspirin for symptomatic intracranial arterial stenosis. N Engl J Med 352: 1305-1316, 2005.

6. Kablak-Ziembicka A, Przewlocki T, Pieniazek P, Musialek P, Tekieli L, Rosławiecka A, Motyl R, Zmudka K, Tracz W and Podolec P: Predictors of cerebral reperfusion injury after carotid stenting: The role of transcranial color-coded Doppler ultrasonography. J Endovasc Ther 17: 556-563, 2010.

7. Park S, Lee DG, Chung WJ, Lee DH and Suh DC: Long-term outcomes of drug-eluting stents in symptomatic intracranial stenosis. Neurointervention 8: 9-14, 2013.

8. Qu H, Li J and Zhao X: Factors affecting pre- and post-stenting computed tomography perfusion in patients with middle cerebral artery stenosis. Exp Ther Med 5: 471-474, 2013.

9. Shi MC, Wang SC, Zhou HW, Xing YQ, Cheng YH, Feng JC and Wu J: Compensatory remodeling in symptomatic middle cerebral artery atherosclerotic stenosis: A high-resolution MRI and microemboli monitoring study. Neurol Res 34: 153-158, 2012.

10. Wang Y, Chen JM, Liu X, Wang J, Li LH, Deng JP and Duan YY: Evaluation of the combined application of ultrasound imaging techniques for middle cerebral artery stent surveillance and follow-up study. PLoS One 8: e79410, 2013.

11. Baaj AA, Agazzi S, Sayed ZA, Toledo M, Spetzler RF and van Loveren H: Surgical management of moyamoya disease: A review. Neurosurg Focus 26: E7, 2009.

12. Abe A, Ueda T, Ueda M, Nogoshi S, Nishiyama $Y$ and Katayama Y: Symptomatic middle cerebral artery stenosis treated by percutaneous transluminal angioplasty: Improvement of cerebrovascular reserves. Interv Neuroradiol 18: 213-220, 2012.

13. Beauchamp NJ Jr, Barker PB, Wang PY and vanZijl PC: Imaging of acute cerebral ischemia. Radiology 212: 307-324, 1999.

14. Małowidzka-Serwińska M, Żabicka M, Witkowski A, Chmielak Z and Deptuch T: Brain perfusion evaluated by perfusion-weighted magnetic resonance imaging before and after stenting internal carotid artery stenosis in asymptomatic and symptomatic patients. Neurol Neurochir Pol 49: 412-420, 2015.

15. Terzis JK, Vekris MD and Soucacos PN: Outcomes of brachial plexus reconstruction 204 patients with devastating paralysis. Plast Reconstr Surg 104: 1221-1240, 1999.

16. Schlötzer W, Huber R and Sehmitz BL: Stent-assisted intraeranial angioplasty: Potentials and Iimitations of pre-and post interventional CT angiography. Rofo 181: 121-128, 2009.

17. Horn P, Lanczik O, Vajkoczy P, Daffertshofer M, Bueltmann E, Werner A, Schmiedek P and Hennerici M: Hemodynamic reserve and high-intensity transient signals in moyamoya disease. Cerebrovasc Dis 19: 141-146, 2005. 
18. Abe A, Ueda T, Ueda M, Nogoshi S, Nishiyama Y and Katayama Y: Recovery of cerebrovascular reserves after stenting for symptomatic carotid artery stenosis. Interv Neuroradiol 16 420-428, 2010

19. Lee M,Zaharchuk G, Guzman R, Achrol A, Bell-Stephens T and Steinberg GK: Quantitative hemodynamic studies in moyamoya disease: A review. Neurosurg Focus 26: E5, 2009.

20. Li Z, Zhou P, Xiong Z, Ma Z, Wang S, Bian H and Chen J: Perfusion-weighted magnetic resonance imaging used in assessing hemodynamics following superficial temporal artery-middle cerebral artery bypass in patients with moyamoya disease. Cerebrovasc Dis 35: 455-460, 2013.

21. Eastwood JD, Lev MH, Wintermark M, Fitzek C, Barboriak DP, Delong DM, Lee TY, Azhari T, Herzau M, Chilukuri VR and Provenzale JM: Correlation of early dynamic CT perfusion imaging with whole-brain MR diffusion and perfusion imaging in acute hemispheric stroke. AJNR Am J Neuroradiol 24: $1869-1875,2003$

22. Schaefer PW, Hunter GJ, He J, Hamberg LM, Sorensen AG, Schwamm LH, Koroshetz WJ and Gonzalez RG: Predicting cerebral ischemic infarct volume with diffusion and perfosion MR imaging. AJNR 23: 1785-1794, 2002.

23. Kajimoto K, Moriwaki H, Yamada N, Hayashida K, Kobayashi J, Miyashita K and Naritomi H: Cerebral hemodynamic evaluation using perfusion-weighted magentic resonance imaging: Comparison with positron emission tomography values in chronic occlusive carotid disease. Stroke 34: 1662-1666, 2003.
24. Ko NU, Achrol AS, Martin AJ, Chopra M, Saloner DA, Higashida RT and Young WL: Magnetic resonance perfusion tracks 133Xe Cerebral blood flow changes after carotid stenting. Stroke 36: 676-678, 2005.

25. Gauvrit JY, Delmaire C, Henon H, Debette S, al Koussa M, Leys D, Pruvo JP and Leclerc X: Dlffusion/Perfusion weighted magnetic resonance imaging after carotid angioplasty and stenting. J Neurol 251: 1060-1067, 2004.

26. Wilkinson ID, Griffiths PD, Hoggard N, Cleveland TJ, Gaines PA, Macdonald S, McKevitt F and Venables GS: Short-term changes in cerebral microhemodynamics after carotid stenting. AJNR Am J Neuroradiol 24: 1501-1507, 2003.

27. Bendok BR, Sherma AK, Hage ZA, Das S, Naidech AM, Surdell DL, Adel JG, Shaibani A, Batjer HH, Carroll TJ and Walker M: Periprocedural MRI perfusion imaging to assess and monitor the hemodynamic impact of intracranial angioplasty and stenting for symptomatic atherosclerotic stenosis. J Clin Neurosci 17: 54-58, 2010. 